\title{
Quantitative characterization of reversible macromolecular associations via sedimentation equilibrium: an introduction
}

\author{
Allen P. Minton ${ }^{1}$ \\ ${ }^{1}$ Section on Physical Biochemistry, Laboratory of Biochemistry and \\ Genetics, National Institute of Diabetes and Digestive and Kidney \\ Diseases, National Institutes of Health, Bethesda MD 20892-0830 \\ USA \\ Corresponding author: Tel, 1-301-496-3604; \\ E-mail, minton@helix.nih.gov
}

Accepted 3 March 2000 regulatory substances, as well as changes in environmental variables such as $\mathrm{pH}$ and ionic strength, affect the relative abundance of various states of association of the interacting macromolecules.

There exist a variety of methods for studying interactions between macromolecules, several of which have recently been reviewed (Srere, 1999). One of the most powerful and versatile techniques for quantitative characterization of reversible protein-protein and proteinnucleic acid interactions in solution is the measurement and analysis of sedimentation equilibrium (Teller, 1973; Minton, 1990). The basic principle underlying sedimentation equilibrium is extremely simple. When a solution containing one or more macromolecular solutes is centrifuged at a constant rotor velocity, each species of macrosolute ultimately attains a time-independent concentration gradient (that is, a dependence of concentration upon distance from the center of rotation) that reflects a balance at all positions between transport of solute due to centrifugal force, and the oppositely directed transport of solute due to back-diffusion (Figure 1). At a given rotor velocity, the gradient depends only upon the buoyant molar mass of that species (i.e., the molar mass of solute reduced by the molar mass of solvent displaced by the solute $)^{1}$ and upon the interactions of that solute species, both attractive and repulsive, with other solutes in the solution. The nature and strength of intermolecular interactions may be deduced by quantitative modeling of experimentally observed gradients and their dependence upon solution composition. Constructing such models in terms of equilibria between postulated complexes is much more straightforward and less ambiguous than modeling of other concentration-dependent properties of a solution. This is due largely to two factors: (1) the simplicity and thermodynamic rigor of the underlying theory, and (2) the ability to calculate unambigously the buoyant mass of each postulated complex from its stoichiometry and the buoyant molar masses of isolated reactants.

Although the basic principles of sedimentation equilibrium have been known for over seventy-five years (Svedberg and Pedersen, 1956), the potential of the method for characterization of equilibrium associations has been by no means fully explored. In the present review we shall

\footnotetext{
${ }^{1}$ For most macrosolutes, which are denser than water, transport due to centrifugal force is outward directed, but lipids and highly lipidated lipoproteins are less dense than water, leading to inwarddirected sedimentation, more commonly referred to as flotation. Macrosolutes less dense than solvent (i.e., water) have negative buoyant masses.
} 


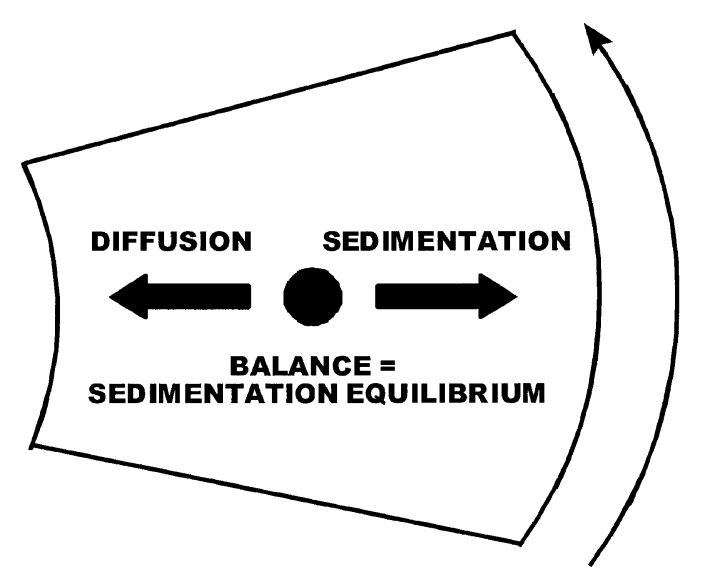

Figure 1. Conditions for sedimentation equilibrium. Solution in a sectorshaped sample cell is being spun in a counter-clockwise direction. The macrosolute represented by a sphere has a density greater than that of solvent, i.e., a positive buoyant molar mass, and is thus subject to an outward-directed sedimenting force.

describe both traditional and non-traditional approaches to the acquisition and analysis of sedimentation equilibrium data, and indicate likely directions for future development.

\section{How do we measure sedimentation equilibrium?}

In order to perform a sedimentation equilibrium experiment it is necessary to be able to measure accurately one or more properties of the solution that are proportional to the weight/volume concentration(s) of macrosolute(s). We shall refer to each such property as a signal. Then one must be able to accurately measure the variation of each signal with radial position (distance from the axis of rotation) after the sample has been spun for a sufficient time for sedimentation equilibrium to be attained. This may be done in two basically different but complementary ways, which we refer to as real-time and post-centrifugation data acquisition respectively (Darawshe and Minton, 1994).

Real-time data acquisition requires the use of a special centrifuge, called an analytical ultracentrifuge, equipped with an optical system that permits an optical signal to be measured as a function of radial distance in a sample cell while the sample is being spun in a special analytical rotor. Analytical ultracentrifuges manufactured by BeckmanCoulter (Fullerton, CA) can measure gradients of UV-visible absorbance (Model XL-A) or absorbance and refractive index (Model XL-I) with satisfactory accuracy and precision (Figure 2).

Post-centrifugation data acquisition is carried out as follows. First, using a conventional swinging bucket rotor in an ordinary preparative ultracentrifuge, the sample is centrifuged to sedimentation equilibrium in a small cylin-

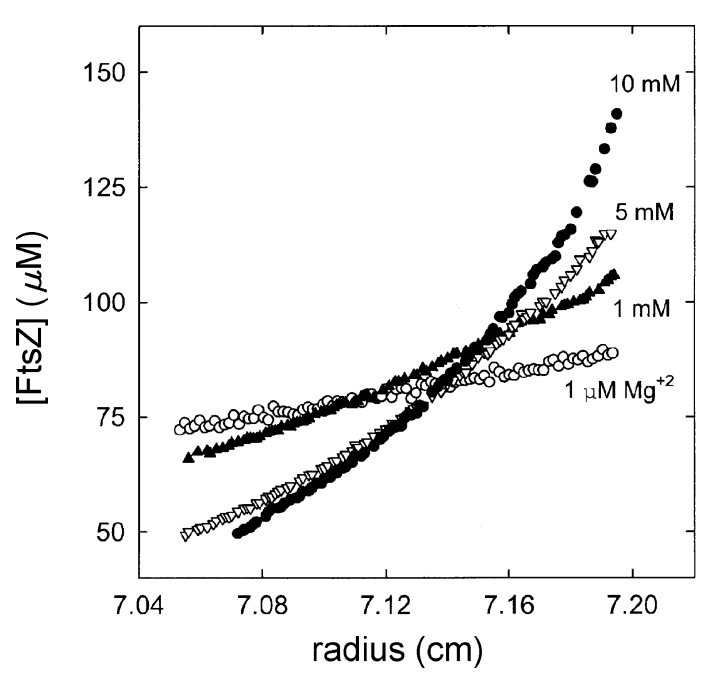

Figure 2. Equilibrium gradients of recombinant bacterial protein FtsZ in solutions containing several concentrations of $\mathrm{Mg}^{2+}$, measured in an analytical ultracentrifuge. Data of (Rivas et al., 2000).

drical tube. Then the centrifuge run is terminated and the centrifuge tube removed from the rotor under conditions such that concentration gradients established at equilibrium remain undisturbed (Attri and Minton, 1986). Then the contents of the tube are sequentially fractionated according to radial position, using an automated centrifuge tube microfractionator (FR-115) manufactured by BRANDEL (Gaithersburg, MD). Finally, the magnitude of any of a variety of possible signals is measured in each fraction using an appropriate assay (Darawshe et al., 1993) (Figure 3).

Both real-time and post-centrifugation data acquisition methods have certain advantages and disadvantages, which have been discussed previously (Darawshe and Minton, 1994). One major advantage of real-time data acquisition is that equilibrium experiments may be completed more rapidly. Hence this approach is likely to be more useful in the study of unstable biomolecules. A major advantage of post-centrifugation data acquisition is the ability it confers on the investigator to select any appropriate assay of solute concentration in each radial fraction, for example, catalytic activity (Osborne et al., 1985) or an immunochemically specific reaction (Liu et al., 1997). ${ }^{2}$

\footnotetext{
${ }^{2}$ One difference between the two methods of data acquisition that may be of particular relevance to potential users of the method in the Asian-Pacific region is the following. The analytical ultracentrifuge is a highly complex and expensive instrument that requires periodic maintenance by manufacturer-trained technicians, who may not be readily available in some countries. In contrast, postcentrifugation data acquisition requires only a simple, inexpensive, maintenance-free centrifuge tube microfractionator in addition to preparative ultracentrifuges of the type already found in almost all biochemistry/molecular biology research facilities.
} 

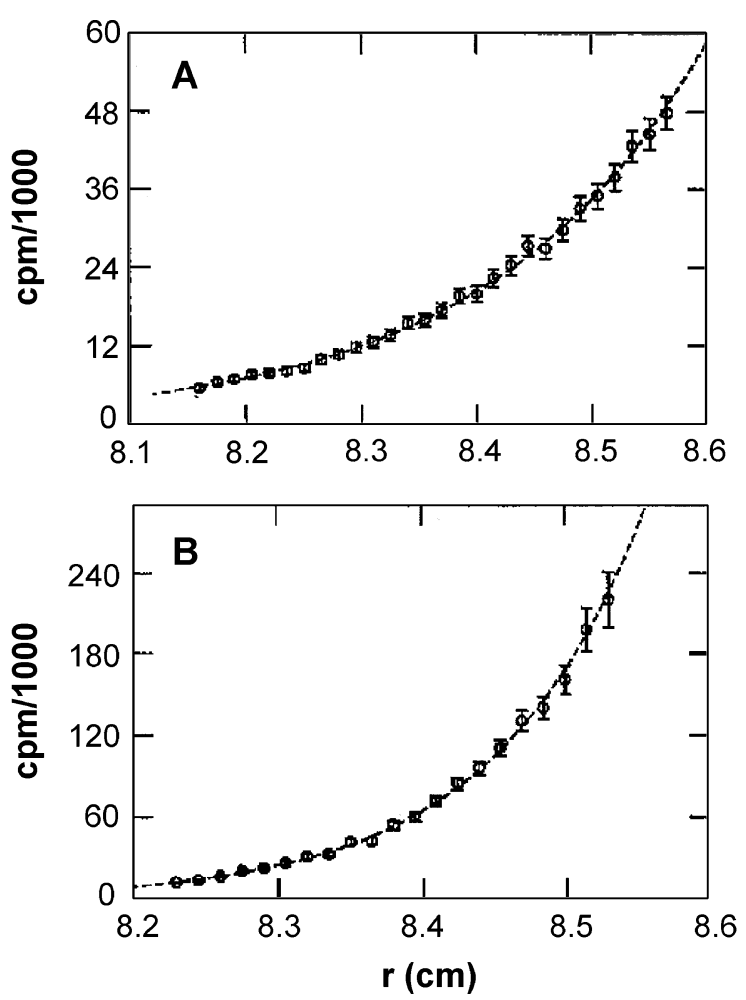

Figure 3. Equilibrium gradients of ${ }^{125}$-labeled ovalbumin (A) and fibrinogen (B), obtained via preparative ultracentrifugation, with subsequent microfractionation and counting of radiolabel in radial fractions. Figure reproduced with permission from (Darawshe et al., 1993).

\section{Analysis of sedimentation equilibrium data}

Let us consider a measurable property of the solution, $\mathrm{S}$, that varies linearly with the weight/volume concentration of each species of sedimentable solute at radial position $\mathrm{r}$ :

$$
S(r)=\sum_{i} S_{i}(r)=\sum_{i} \alpha_{i} w_{i}(r)
$$

where $w_{i}$ and $\alpha_{i}$ denote respectively the weight/volume $(w / v)$ concentration and signal proportionality constant of the th solute species. Some examples of suitable properties are the uv or visible absorbance or refractive index at a particular wavelength of light, or the radioactivity of a radiolabeled solute. ${ }^{3}$ When the solution is sufficiently dilute in macromolecular solutes so that repulsive interactions between macrosolute molecules may be neglected (typically $<5-10 \mathrm{mg} / \mathrm{ml}$ total macrosolute in solutions of moderate ionic strength), it can be rigorously shown (Hsu and Minton, 1991; Rivas et al., 1999) that at sedimentation equilibrium, the radial dependence of signal $\mathrm{S}$ is given by

\footnotetext{
${ }^{3}$ In general, a "suitable" property is one that can be shown to satisfy the condition that $S_{i}(r)$ depends only on $w_{i}(r)$; i.e., is independent of $w_{j}(r)$ for all $j \neq i$.
}

$\frac{d \ln S(r)}{d r^{2}}=\frac{M_{S}^{*}(\{w(r)\}) \omega^{2}}{R T}$

where $r$ denotes the distance from the center of rotation, $\{W(r)\}$ the composition of solute species at radial position $r, \omega$ the angular velocity of the rotor, $R$ the molar gas constant, $T$ the absolute temperature, and $M_{S}^{*}$ is the signal-average buoyant molar mass, given by

$$
M_{S}^{*}(\{w(r)\})=\frac{\sum_{i} \alpha_{i} w_{i}(r) M_{i}\left(1-\bar{v}_{i} \rho_{o}\right)}{\sum_{i} \alpha_{i} w_{i}(r)}
$$

where $M_{i}$ and $\bar{v}_{i}$ are respectively the molar mass and partial specific volume of the th solute species. ${ }^{4,5}$ For any given model scheme for self- or hetero-association, the value of $M_{S}^{*}$ may be calculated as a function of the total concentrations of all chemically distinct components, the buoyant molar masses of each component (as monomer), and the equilibrium association constants describing each postulated association (Teller, 1973; Hsu and Minton, 1991; Rivas et al., 1999). A simple example of such a model is presented in the Appendix.

The nature of the associations underlying the experimentally observed sedimentation behavior is elucidated by attempting to fit the experimentally observed dependence of $M_{S}^{*}$ upon solution composition by various functional forms of equation 3 calculated according to a variety of models for association. ${ }^{6}$ Two strategies for accomplishing this objective are illustrated in Figure 4. If a particular model, with a particular set of association equilibrium constants can account for the observed dependence to within experimental precision, then the data are said to be consistent with that model. If more than one model is capable of fitting the data to within experimental precision, then additional experiments are

\footnotetext{
${ }^{4}$ The partial specific volume of the th species is defined as the derivative of solution volume with respect to $\mathrm{w} / \mathrm{v}$ concentration of that species. It may be independently measured, or, in many cases, estimated to a very good approximation from the chemical composition of the species (Durschlag, 1986).

${ }^{5}$ The interpretation of $\mathrm{M}_{\mathrm{S}}^{*}$ in more concentrated solutions requires consideration of repulsive (nonideal) interactions between molecules of macrosolute, in addition to attractive interactions leading to the formation of equilibrium complexes (Rivas et al., 1999).

${ }^{6}$ The equilibrium data which are modeled may be either the dependence of $\mathrm{M}_{\mathrm{S}}^{*}$ upon solution composition, derived from the original gradients $S(r)$ by means of equation 2 , or the actual gradients themselves, through combination of equation 2 with the integrated form of equation 3 . A variety of personal computer software programs for performing both types of modeling procedure is freely available, either from the software library of the Interest Group for Reversible Associations in Structural and Molecular Biology (RASMB) at www.bbri.org/rasmb/rasmb.html, or from the author upon request.
} 

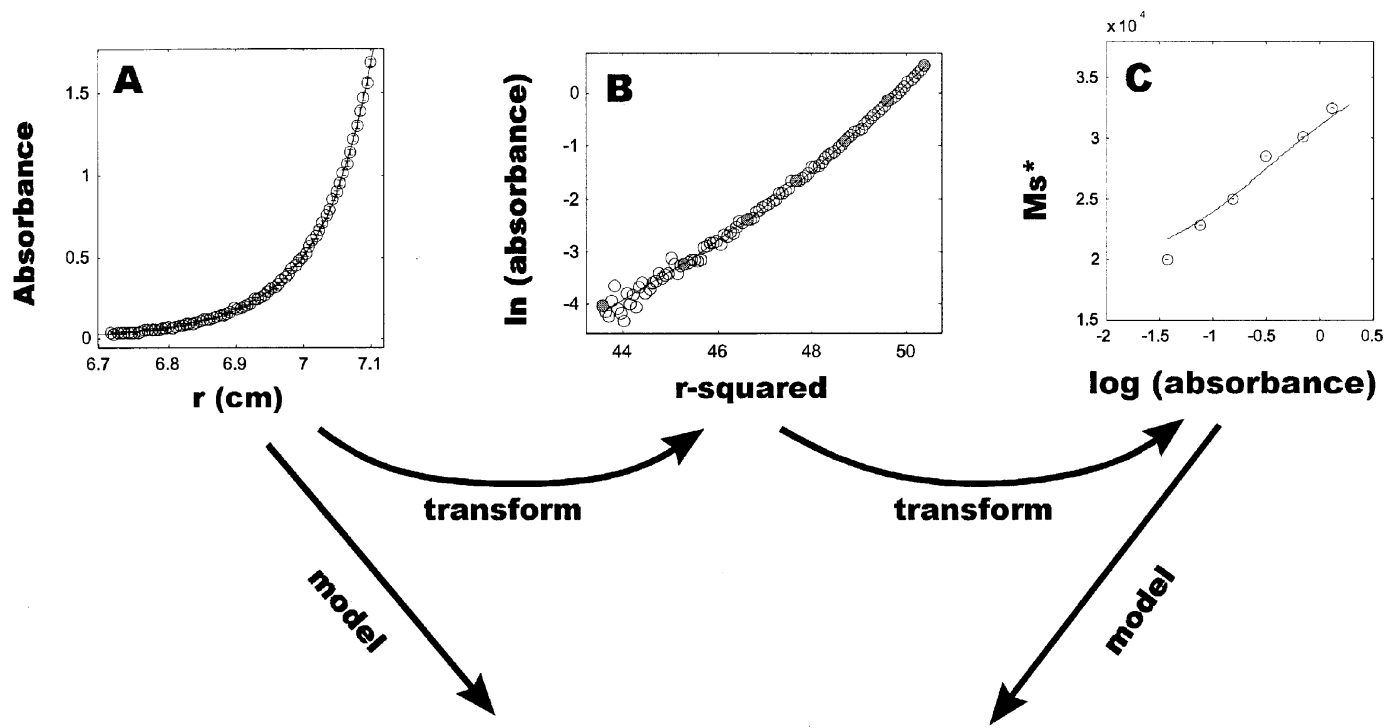

$\log$ (absorbance)

association stoichiometry, equilibrium constants

Figure 4. Two strategies for analysis of sedimentation equilibrium. Strategy I: Raw data $\{r, S\}(P a n e l A)$ is transformed to $\left\{r^{2}\right.$, In $\left.S\right\}$ (Panel B). Local slope and amplitude of $S$ leads via text equation 2 to $\left\{\log S, M_{S}^{*}\right\}$ (Panel $C$ ), which may be fitted by an association model to yield parameters of association. Strategy II: Raw data (Panel A) is fitted directly by a compound association model that yields an analytically integrated form of text equation 2 .

required to discriminate between alternative models (Hsu and Minton, 1991).

\section{Conclusion}

Recent developments in instrumentation and processing of data have greatly increased the variety of scientific questions which may be studied and successfully answered via measurement and analysis of sedimentation equilibrium. Perhaps the most empowering of these developments are the use of tracers and the measurement of non-optical in addition to optical measures of solute concentration. Examples of the application of these methods may be found in Rivas et al. (1992), Laue et al. (1993), and Rivas et al. (1994). In combination, these techniques permit sedimentation equilibrium measurements to be carried out over an unprecedentedly broad range of solution compositions, making possible the quantitative characterization of associations that are manifested over a range of nanomolar to millimolar concentrations of solute. Access to such a broad range of compositions is essential to an understanding of the functional energetics of ternary or larger biomolecular complexes involving multiple modes of association.

\section{Appendix}

Modeling sedimentation equilibrium of a self-associating macrosolute.

To illustrate the use of sedimentation equilibrium as a means of characterizing reversible association in solution, we treat explicitly the simple case of a single macrosolute that can exist as an equilibrium mixture of monomer and dimer. Let the equilibrium constant be denoted by $\mathrm{K} \equiv \mathrm{w}_{2} / \mathrm{w}_{1}{ }^{2}$, where $\mathrm{w}_{\mathrm{i}}$ is the weight/volume concentration of i-mer. For a single solute component, $\alpha_{1}=\alpha_{2}=\alpha$ (i.e., $S=\alpha \mathrm{W}_{\text {tot }}$ ), and $\bar{v}_{1}=\bar{v}_{2}=\bar{v}$. For this case, text equation 3 reduces to

$M_{S}^{*}=M_{1}^{*} \frac{w_{1}+2 * w_{2}}{w_{1}+w_{2}}=M_{1}^{*}\left[w_{1}+2 K w_{1}^{2}\right] / w_{t o t}$

where $M_{1}^{*}$ denotes the buoyant molar mass of monomer $\left[\mathrm{M}_{1}(1-\bar{v} \rho)\right]$, and $\mathrm{w}_{\text {tot }}$ the total $\mathrm{w} / \mathrm{v}$ concentration of macrosolute. By conservation of mass, $w_{\text {tot }}=w_{1}+w_{2}=w_{1}+$ $\mathrm{Kw}_{1}{ }^{2}$, which can be solved for the concentration of monomer as a function of $\mathrm{K}$ and $\mathrm{w}_{\text {tot: }}$ :

$w_{1}=\left[-1+\left(1+4 K w_{t o t}\right)^{1 / 2}\right] / 2 K$

Thus, for any specified values of $\alpha, M_{1}^{*}$ and $\mathrm{K}$, one can use equations $A 1$ and $A 2$ to solve for $M_{S}^{*}$ as a function of $\mathrm{S}$. The solid curve in panel $\mathrm{C}$ of Fig. 4 was calculated using equations $A 1$ and $A 2$ (with $\alpha=1$ ) together with the values of $M_{1}^{*}$ and $\mathrm{K}$ obtained by least-squares fitting of this model to the indicated data.

\section{References}

Alberts, B. (1998) The cell as a collection of protein machines: preparing the next generation of molecular biologists. Cell. 92: 291-294

Attri, A. K. and Minton, A. P. (1986) Technique and apparatus 
for automated fractionation of the contents of small centrifuge tubes: application to analytical ultracentrifugation. Analytical Biochemistry. 152: 319-328

Darawshe, S. and Minton, A. P. (1994) Quantitative characterization of macromolecular associations in solution via real-time and postcentrifugation measurements of sedimentation equilibrium: A comparison. Anal. Biochem. 220: 1-4

Darawshe, S., Rivas, G. and Minton, A. P. (1993) Rapid and accurate microfractionation of the contents of small centrifuge tubes: application in the measurement of molecular weights of proteins via sedimentation equilibrium. Anal. Biochem. 209: $130-135$

Durschlag, H. (1986) Specific volumes of biological macromolecules and some other molecules of biological interest. In Hinz, H.-J., ed. Thermodynamic Data for Biochemistry and Biotechnology. Springer, Berlin. 45-128

Hsu, C. and Minton, A. P. (1991) A strategy for efficient characterization of macromolecular heteroassociations via measurement of sedimentation equilibrium. J. Mol. Recog. 4: 93104

Laue, T., Senear, D., Eaton, S. and Ross, J. (1993) 5-Hydroxytrptophan as a new intrinsic probe for investigating proteinDNA interactions by analytical ultracentrifugation. Study of the effect of DNA on self-assembly of the bacteriophage lambda cl repressor. Biochemistry. 32: 2469-2472

Liu, J., Reitz, B., Fox, J. and Shire, S. J. (1997) Determination of the average molecular weights of antibody and its complexes in serum using a preparative centrifuge. Pharm. Res. 14: S348

Minton, A. P. (1990) Quantitative characterization of reversible molecular associations via analytical centrifugation. Anal. Biochem.
190: $1-6$

Osborne, J. C., Bengtsson, G., Lee, N. S. and Olivecrona, T. (1985) Studies on inactivation of lipoprotein lipase: role of the dimer to monomer dissociation. Biochemistry. 24: 5606-5611

Rivas, G., Fernandez, J. A. and Minton, A. P. (1999) Direct observation of the self-association of dilute proteins in the presence of inert macromolecules at high concentration via tracer sedimentation equilibrium: theory, experiment, and biological significance. Biochemistry. 38: 9379-9388

Rivas, G., Ingham, K. C. and Minton, A. P. (1992) $\mathrm{Ca}^{2+}$-linked self-association of human complement $\mathrm{C} 1 \mathrm{~s}$. Biochemistry. 31 : 11707-11710.

Rivas, G., Ingham, K. C. and Minton, A. P. (1994) Ca $\mathrm{Ca}^{2+}$-linked association of human complement $\mathrm{C} 1 \mathrm{~s}$ and $\mathrm{C} 1 \mathrm{r}$. Biochemistry. 33: $2341-2348$

Rivas, G., Lopez, A., Mingorance, J., Ferrandiz, M. J., Zorrilla, S., Minton, A. P., Vicente, M. and Andreu, J. M. (2000) Magnesiuminduced linear self-association of the FtsZ bacterial cell division protein monomer. The primary steps for FtsZ assembly. Biochemistry (in press).

Rivas, G., Stafford, W. and Minton, A. P. (1999) Characterization of heterologous protein-protein interactions using analytical ultracentrifugation. Methods. 19: 194-212.

Srere, P. A., ed. (1999) Special issue: Protein-protein interactions. Methods. 19: 191-349.

Svedberg, T. and Pedersen, K. O. (1956) The Ultracentrifuge. Johnson Reprint Corp., New York. 473 pp.

Teller, D. C. (1973) Characterization of proteins by sedimentation equilibrium in the analytical ultracentrifuge. Methods in Enzymology. 23: 346-441 